\title{
SÉANCE DU 8 NOVEMBRE 1918
}

\author{
PrésIdENGE DE M. P.-A. DANGEARD.
}

Lecture est donnée du procès-verbal de la dernière séance, dont la rédaction est adoptée.

M. le Président annonce le décès de M. Casimir de Candolle et celui du fils de notre confrère M. Molliard, au cours d'un congé de convalescence.

Il est ensuite donné connaissance du travail suivant de M. Vuillemin.

\section{Le placenta. Son indépendance primitive}

\section{Par M. Paul Vuillemin.}

Dans une Note antérieure ${ }^{1}$, j'ai considéré le carpelle comme un membre mixte, composé de deux parties concrescentes, un phylloïde et un frondoïde; la paroi ovarienne appartient à la première, le placenta à la seconde.

Cette glossologie n'exprime pas clairement ma pensée. Le terme membre mixte ne fait pas suffisamment ressortir la dualité originelle des deux sortes de membres associés habituellement. dans le pistil; il s'agit, non des parties d'un membre mixte, mais de deux membres essentiellement différents lors même qu'ils sont concrescents. L'un est homologue de la feuille, l'autre de la fronde, sans présenter pourtant le mode particulier de différenciation évoqué par ces mots. Il existe un terme technique applicable à tout appendice homologue de la feuille. Phyllome est un nom générique embrassant plusieurs espèces, entre autres, feuilles, phylloïde de Lignier, carpelle. Ce dernier nom

1. Vullummin (P.), Le Placenta. Sa nature ligulaire (Bull. Soc. bot. de France, LXII, p. 42-49, 1915.) 


\section{$2 \mathrm{BHL}$ Biodiversity Heritage Library}

1918. "Séance Du 8 Novembre 1918." Bulletin de la Société botanique de France 65, 144-144. https://doi.org/10.1080/00378941.1918.10836066.

View This Item Online: https://www.biodiversitylibrary.org/item/8685

DOI: https://doi.org/10.1080/00378941.1918.10836066

Permalink: https://www.biodiversitylibrary.org/partpdf/159710

\section{Holding Institution}

Missouri Botanical Garden, Peter H. Raven Library

\section{Sponsored by}

Missouri Botanical Garden

\section{Copyright \& Reuse}

Copyright Status: Public domain. The BHL considers that this work is no longer under copyright protection.

This document was created from content at the Biodiversity Heritage Library, the world's largest open access digital library for biodiversity literature and archives. Visit BHL at https://www.biodiversitylibrary.org. 